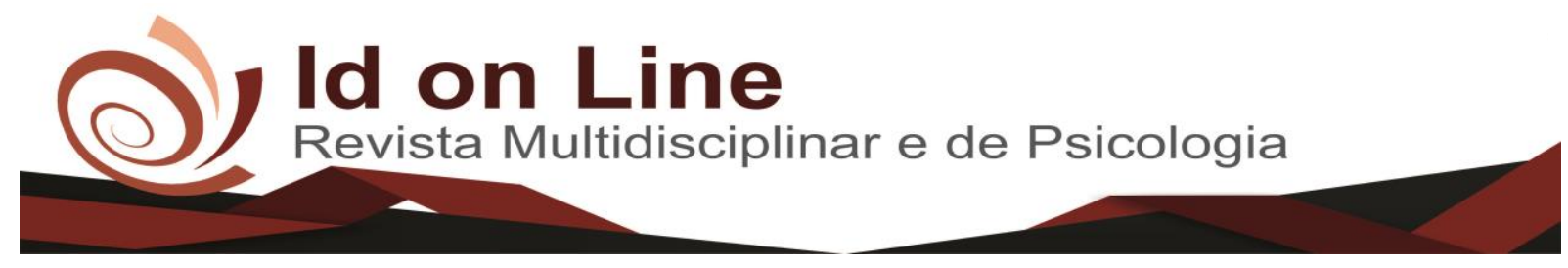

Artigo de Revisão

\title{
Tratamento de mordida aberta anterior: Uma revisão de literatura
}

\author{
Islana Cleia Carvalho Vieira ${ }^{1}$; Idalécio Carvalho Vieira ${ }^{2}$; \\ Morgana Souza de Azeredo ${ }^{3}$; Lauren Stephanie Vieira Jardim ${ }^{1}$; Jônatas Pereira do Prado ${ }^{1}$; \\ Jade Alexandre Belo Reis ${ }^{l}$; Anne Maria Guimarães Lessa ${ }^{4}$
}

\begin{abstract}
Resumo: A mordida aberta anterior é um problema de oclusão de etiologia complexa e que ao longo do tempo foi sendo tratada devido a evolução e ao avanço dos tratamentos ortodônticos que acrescentaram de forma crucial a aplicabilidade do cuidado em muitas situações, mas para isso o ortodontista deve compreender cada situação em si, diagnosticar de forma correta e analisar as propriedades de cada tratamento para que os riscos e limitações sejam eliminados. E, neste sentido, este estudo objetiva fazer uma análise do tratamento de mordida aberta anterior (MAA), por meio de uma revisão de literatura. Para isso foi utilizado o método qualitativo, trazendo uma revisão de literatura abrangendo estudos das bases de dados do Scielo, Pubmed, Medline e Lilacs, sendo selecionados os estudos do idioma português e inglês entre os anos de 2010 a 2018. Diante disso, busca-se trazer um melhor entendimento sobre o assunto, trazendo um arcabouço teórico para profissionais da saúde sobre a situação exposta, destacando os tratamentos mais usados em pacientes com problemas de oclusão que podem ter resultados bastante satisfatórios.
\end{abstract}

Descritores: Aplicação. Mordida Aberta Anterior. Oclusão. Ortodontia. Tratamento.

\section{Treatment of anterior open bite: A literature review}

\begin{abstract}
The anterior open bite is an occlusion problem of complex etiology that has been treated over time due to the evolution and advancement of orthodontic treatments that have added crucially the applicability of care in many situations. For this reason, the orthodontist must understand each situation in order to correctly diagnose and analyze the properties of each treatment so that the risks and limitations are eliminated. Therefore, this study aims to make an analysis of the treatment of anterior open bite (MAA), through a literature review. For this purpose, it was used the qualitative method and a literature review covering studies of the databases of Scielo, Pubmed, Medline and Lilacs, in which studies in Portuguese and English were selected between the years of 2010 and 2018. In order to bring a better understanding on the subject, a theoretical framework for health professionals about the exposed situation was developed to highlight the treatments most used in patients with occlusion problems with have quite satisfactory results.
\end{abstract}

Descriptors: Application. Anterior Open Bite. Occlusion. Orthodontics. Treatment.

\footnotetext{
${ }^{1}$ Graduanda do curso de Odontologia da Faculdade Independente do Nordeste. Contato: islana_carvalho@hotmail.com

${ }^{2}$ Cirurgião-Dentista. Graduado em Odontologia pela Univale (MG). Pós graduação em Ortodontia. Especialista em Ortodontia e Ortopedia funcional dos maxilares. Pós graduação em cirurgia oral menor. Especialista em Implantodontia. Mestrando em Implantodontia (SLMandic Campinas).

${ }^{3}$ Cirurgiã-Dentista. Graduada pela Fainor-VCA. Pós Graduação em endodontia pela ABO-VCA. Mestranda em Odontopediatria - SLMandic Campinas.

${ }^{4}$ Mestra e professora do curso de Odontologia da Faculdade Independente do Nordeste.
} 


\section{Introdução}

A mordida aberta anterior (MAA) é um tipo de má oclusão onde há trespasse vertical negativo existente entre as bordas incisais superiores e inferiores, e, este problema, é imerso em muitos desafios no que diz respeito ao seu tratamento, sendo um problema de etiologia complexa que relaciona o sistema esquelético, dentário, fatores respiratórios, neurológicos ou relacionados aos hábitos cotidianos ${ }^{1,2}$.

Vale salientar, ainda, que este tipo de patologia dentária gera grande comprometimento estético e funcional e, geralmente, afeta pessoas mais jovens e devido a discrepância no sentido vertical permite menor estabilidade nos resultados finais ${ }^{3}$.

Nos estudos que abordam a MAA e seu tratamento é comum encontrar informações controversas e discordantes $^{3}$, principalmente sobre a etiologia e possíveis tipos de tratamentos, não havendo consenso a respeito. Destaca-se que a maioria dos estudos define a MAA como a ausência de contato incisal dos dentes anteriores em relação cêntrica ${ }^{4,5}$.

A prevalência da MAA é muito variável conforme visto nos estudos sobre o tema e depende da corrente ou posição seguida pelos autores dos trabalhos realizados. Em um estudo feito por Artese et al. (2011) houve uma prevalência na população entre $1,5 \%$ e $11 \%$, destacando a preocupação na qual o tratamento deva ser feito da melhor forma, permitindo uma correção efetiva e estável ${ }^{4}$. Entretanto, no estudo de Oliveira (2015) taxas de prevalência para a MAA variaram de 2 a $8 \%$ pautado em estudos em crianças pré-escolares ${ }^{5,6}$.

$\mathrm{Na}$ aplicação do tratamento de MAA assim como na mordida cruzada posterior (MCP) e na sobressaliência, o Cirurgião-Dentista deve se pautar nas evidências científicas de acordo com o tipo de movimentação ou efeito desejado já que todo tratamento deve estar adequado a cada situação e paciente para que os resultados sejam individualizados e eficientes ${ }^{\text {-14 }}$.

Diante disso, avaliar a propriedades de cada tratamento e situação peculiar do paciente, bem como suas vantagens, desvantagens, indicações e limitações se faz determinante, por isso que a degradação do material ou da força devido à movimentação, bem como a escolha do elástico sintético e outras características tem que ser consideradas ${ }^{11}$.

Diante disso, esse estudo busca ser ferramenta de apoio para o tratamento da MAA, descrevendo os resultados de estudos e relatos de casos de tratamentos realizados para esta 
situação. Diante disso é justificável tratar deste tema pelo fato de que é possível perceber que, embora existam poucos estudos recentes relacionando ao tratamento dessa má-oclusão, estes trazem implicações clínicas positivas ao paciente, melhorando o problema vertical, estético e, consequentemente, melhorando a qualidade de vida destes.

O objetivo deste trabalho é trazer uma discussão a partir de uma revisão de literatura sobre o tratamento de MAA e para isso foram consultados artigos científicos como base para uma revisão bibliográfica, realizada tendo por base teórica artigos de revistas científicas presente nas bases de dados do Scielo, Pubmed, Medline e Lilacs.

Como critérios de inclusão na seleção dos artigos foram selecionados pelos títulos nos descritores, completos ou em parte as palavras: mordida aberta anterior, tratamento da MAA, que estivessem no idioma português e inglês e trabalhos disponíveis gratuitamente e na íntegra. Quanto aos critérios de exclusão considerou-se estudos datados de mais de 8 anos e que fossem documentos do tipo livro, capítulos de livro, artigos de opinião ou artigos que não abordaram a má-oclusão, foram localizadas o total de 21 referências, entre período de 2010 a 2018.

\section{Revisão de Literatura}

Sem dúvida que o tratamento da MAA vem a ser um dos desafios da má- oclusão e muitos profissionais que trabalham com este buscam interceptar esta má- oclusão já em idade precoce. Este tipo de má-oclusão vertical se desenvolve como resultado da interação de muitos fatores etiológicos diferentes, incluindo chupar o polegar e o dedo, hábitos nos lábios e na língua, vias respiratórias obstrução e anormalidades de crescimento esquelético verdadeiras ${ }^{12}$, 13 .

O tratamento para mordida aberta (MA) varia de observação ou simples controle de hábito para procedimentos cirúrgicos complexos. Uma identificação da etiologia e diagnóstico precoce melhoram as chances do sucesso do tratamento ${ }^{6,14}$

Os Cirurgiões-Dentistas que atuam no tratamento da MAA devem conhecer todas as nuances, uso de força e medidas a serem tomadas, salientando que o tratamento dessa máoclusão deve ser precoce para gerar resultados mais eficientes. Geralmente, devido à discrepância óssea, utiliza-se elásticos intermaxilares, sem extrações e sem intervenção 
cirúrgica, buscando intervir na relação anteroposterior oclusal, melhorando o equilíbrio oclusal $^{9-11}$.

É importante acrescer que clinica e cefalometricamente os pacientes com MAA têm um aumento do terço inferior da face e que tipos faciais verticais poderiam ser separados de maneira confiável usando medidas extraorais para homens e mulheres. Sendo que a classificação clínica vertical dos pacientes pode ser realizada, mas deve ser tentado com cuidado e treinamento apropriado $^{15}$.

Uma vez identificada a anormalidade esquelética, o paciente pode ser classificado como mordida aberta dentária ou oclusão normal. Pacientes com aumento da altura facial inferior podem ou não tem uma MAA dentária. Em todos os pacientes, uma MA existe durante a troca de incisivos permanentes, que faz parte do crescimento e desenvolvimento ${ }^{12,16}$.

Em resumo, tanto esqueleto normal quanto longo como morfologia são observadas em associação com e oclusão dentária de MA. Em outras palavras, a oclusão dentária por MA não é indicativa de relação esquelética ${ }^{17}$.

Dentistas e Fonoaudiólogos geralmente atribuem que o tratamento deve ser feito conjuntamente, avaliando a dentição, má-oclusão e o comportamento respiratório que deve fornecer monitoramento contínuo de sucessivos ciclos respiratórios, medir tanto a inspiração, fluxo de ar expiratório, fornecer medições simultâneas de fluxo de ar oral e nasal, interferir minimamente com comportamento respiratório normal, e ter um alto grau de confiabilidade e reprodutibilidade ${ }^{6,9}$.

Afirmar que não existirão recidivas para a má-oclusão de mordida aberta é complicado. Indicar ao paciente que algumas abordagens são mais racionais do que os outros é justo. Infelizmente, os resultados clínicos a longo prazo não são bem documentados. O tratamento para problemas de MA varia de observação ou simples procedimentos de controle de hábitos a procedimentos cirúrgicos complexos ${ }^{18}$.

Tal situação é complicada pelo fato de que o crescimento vertical é a última dimensão para ser concluído e significa que, às vezes um tratamento simples prevalecerá, enquanto em outras pode parecer ser bem sucedido, mas pode ocorrer recidiva. Isso também implica que alguns tratamentos podem ser prolongados, mesmo se tratados precocemente, mas não removidos os fatores etiológicos ${ }^{2,8}$. 
A relação custo-eficácia esses planos demorados devem ser questionados. As técnicas de tratamento podem ser categorizadas como hábito, aparelho ou cirúrgico. Técnicas simples são aquelas em que o fator etiológico é removido e a mordida se fecha pelo processo eruptivo normal, ou o fechamento é aprimorado usando aparelhos ortodônticos. Procedimentos mais difíceis são aqueles em que a intrusão (ativa ou relativa intrusão alcançada pela inibição da erupção dos dentes posteriores) é tentada com aparelhos ortodônticos ${ }^{18,19}$.

O trespasse vertical negativo que atinge a região anterior nos casos de MAA pode afetar a mastigação, fonação, a estética e, consequentemente, a autoestima e qualidade de vida do paciente, pois em muitos casos as alterações morfológicas podem ser significativas ${ }^{6}$. Logo, com relação ao tratamento ortodôntico, para a MAA, a literatura cita o uso de aparelhos extrabucais, e "bite-blocks", primando pela redução e correções da MAA ${ }^{7}$. Este tratamento precoce é muito viável, uma vez que a direção do crescimento pode ser controlada e evitar um tratamento mais agressivo $^{8}$.

O uso de elásticos também vem sendo cada vez mais fomentados na área de Ortodontia, destacando vantagens para a movimentação dentária, tanto na retração de dentes e fechamento de espaços, bem como na correção de relações interarcos e também como auxiliares na utilização de aparelhos extrabucais ${ }^{9,10}$. Os elásticos ortodônticos são ferramentas importantes para que se obtenham resultados favoráveis no tratamento ortodôntico ${ }^{10}$.

Em alguns casos, a cirurgia ortognática é a última a si recorrer. Muitas vezes as abordagens de tratamento são combinadas, principalmente em casos em que a quando a etiologia não é diagnosticada ${ }^{8}$.

Para situações em que a MAA foi gerada por hábitos de sucção em criança, se não ocorrer a autocorreção deve-se fazer o controle dos hábitos deletérios e a atenção multiprofissional aliada ao Fonoaudiólogo, Otorrinolaringologista e Ortodontista Em crianças pequenas controlar o hábito por si só pode ser suficiente para permitir que os dentes entrem em erupção para uma posição normal. O tratamento deve começar quando o benefício para o paciente supera os riscos (odontológico, emocional e psicológico) da interrupção do hábito ${ }^{18,}$ 19

Melink et al. ${ }^{15}$ dita ainda que geralmente incidem em má-oclusão de MCP, MAA e sobressaliência, e destaca que a incidência é mais frequente em crianças, devido aos fatores de 
sucção e amamentação envoltos no processo, e destaca que a MCP advém também destes hábitos.

Rochele et al. ${ }^{16}$ ditam de igual forma que entre as causas de más-oclusões os hábitos deletérios são bastante frequentes, e devem ser diagnosticados precocemente para que não ocasione problemas maiores, por isso enfatiza o diagnóstico dos hábitos nocivos como crucial e determinante para um tratamento de sucesso, evitando inclusive agravos ao sistema estomatognático.

Bona et al. ${ }^{17}$ destaca que a MAA é dentre as oclusopatias a que traz um comprometimento estético funcional mais latente e afeta alimentação e ainda na pronúncia de fonemas, afetando a qualidade de vida, por isso é salutar interpor o tratamento para gerar melhores condições funcionais e estéticas com uma abordagem preventiva e por isso incentivase associar os Ortodontistas e Fonoaudiólogos, no tratamento ainda incentivando em algumas situações o acompanhamento psicológico especializado.

Artese et al. (2011) ${ }^{4}$ destaca que há outras ferramentas para auxiliar a corrigir hábitos funcionais usando mecanismos que impeçam que a língua se escore sobre os dentes. As pontas ativas ou esporões compelem uma mudança na localização de repouso da língua, garantindo que os dentes irrompam e a MA seja corrigida.

O uso de aparelhos centra-se em vários objetivos: impedir a erupção dentária e, assim, controlar o desenvolvimento, para reduzir ou redirecionar o esqueleto e ajudar no crescimento com forças intra-orais ou extra-orais, ou para extrusão dentes anteriores. Blocos de mordida são usados como um componente de aparelhos ortodônticos Bite Blocks para interferir, contar ou controlar a erupção dos dentes posteriores ${ }^{18}$.

Na correção de problemas de MA esquelética, pode-se citar ainda conforme estudos feitos o Binator de Balters fechado que é tido como uma espécie de ativador, bionatores ou reguladores (a maioria com a inclusão da mordida posterior blocos), têm sido utilizados para controle do crescimento da dentição mista, resolvendo problemas de interposição lingual ou sucção ${ }^{19}$.

Outra abordagem de aparelhos usa dispositivos extra-orais para impedir o padrão de crescimento esquelético e dental vertical, como um casquete de tração alta. O maior problema com o casquete é que é quase impossível obter uma força vertical pura ${ }^{20}$. 
Rahpeyma et al. (2014) ${ }^{11}$ sugere controlar a força vertical usando forças na região posterior da mandíbula por aparelho extra-bucal, que pode ser útil na redução dos aumentos da altura molar ganhando controle do ângulo do plano oclusal. Quando os pacientes têm aumento do desenvolvimento vertical e uma má-oclusão de classe II, existe o potencial para usar em combinação com um aparelho funcional incorporando blocos de mordida posterior.

Aparelhos de fio reto e nivelamento dos arcos podem corrigir espontaneamente MA leves, tendo mais eficácia se não houver nivelamento abusivo do arco inferior, pois nesses casos abre a mordida e é contra-indicado ${ }^{9,21}$.

Alguns casos de MA podem ser tratados passando-se os arcos, associado ao uso de elásticos intermaxilares para fechar a mordida combinada com o uso de elásticos verticais. $\mathrm{O}$ NiTi retangular de fios e elásticos para fechar uma MAA vem sendo útil nesses casos e muitos cuidados devem ser tomados para não erupcionar os dentes extensivamente quando paciente tem a altura facial aumentada ${ }^{19,21}$.

Em resumo, qualquer abordagem de dentição mista deve levar em consideração vários fatores e a terapia fixa com uso de aparelho com sua biomecânica extrusiva, não deve reverter os ganhos feitos anteriormente. Combinar técnicas é importante e podem ser essenciais mesmo durante as fases de acabamento e retenção ${ }^{17,21}$.

Por esse motivo, pode ser melhor lidar apenas com problemas leves ou moderados ou aqueles em pacientes que estão perto do fim do crescimento, do que problemas graves de MA. Em segundo lugar, qualquer tratamento destinada a controlar a erupção em um arco deve guardar compensar ou controlar a erupção compensatória no arco oposto ${ }^{6,12}$.

Entre os tratamentos está o manejo cirúrgico que é a técnica de correção feita por cirurgia e que visa extrair segundos e / ou terceiros molares, se forem os únicos fonte de contatos centrais. As glossectomias foram usadas para corrigir problemas de MA associados hábitos anormais de língua. Sua eficácia no fechamento problemas de MAA ou posterior ainda não estão fundamentados na literatura ${ }^{4,8}$.

Procedimentos cirúrgicos sempre devem ser feitos com toda cautela, mesmo que o reposicionamento superior da maxila é uma das cirurgias ortognáticas mais estáveis. Vale salientar no tratamento a importância de acompanhamento com um Otorrinolaringologista confiável, Fonoaudiólogo e outros profissionais necessários para ajudar nesta situação, salientando que há casos onde há obstrução da via aérea nasal ${ }^{18-21}$. 


\section{Discussão}

Nota-se, portanto que discutir o tratamento ortodôntico em casos de MAA é importante por haver vários tipos de tratamento e pouca discussão teórica sobre o tema. Estudos feitos sobre a temática abordada destacam que a eliminação dos hábitos deletérios é necessária em alguns casos e a necessidade de haver reforços positivos, dos pais, familiares e dos profissionais envolvidos, trazendo auto estima e apoio para criança e para isso a relação profissional-paciente deve ser muito bem estruturada, criando um vínculo que ajudará a trazer um bom prognóstico do tratamento efetuado ${ }^{6,8,14,18,21}$

Romero et al. (2011) por sua vez relaciona os hábitos deletérios a sua prevalência na maioria dos casos e destaca que este é bastante incidente desde sempre e dá destaque aos hábitos de sucção sem fins nutritivos, que geralmente são os primeiros maus hábitos da pessoa, instalado nas primeiras fases da vida ${ }^{19}$.

Miotto et al. (2014) ${ }^{2}$ e Artese et al. (2011) ${ }^{4}$ em estudo feito destacam a necessidade de uma atenção profissional multidisciplinar e cuidado minucioso, este mesmo autor cita como hábitos deletérios, a projeção da língua; sucção de dedos e lábios, deglutição atípica; e sucção de chupeta como fatores que podem gerar MAA e estão inclusas no tratamento que deve-se tratar os hábitos.

Estudo feito por Rocha e Rodrigues $(2017){ }^{7}$ dizem que os hábitos deletérios geralmente incidem em más-oclusões de MCP, MAA e sobressaliência, e destaca que a incidência é mais frequente em crianças, devido aos fatores de sucção e amamentação envoltos no processo, e destaca que a MCP advém também destes hábitos. Mas pode ocorrer também em adultos e o uso de aparelhos pode ser útil e eficaz para evitar a erupção dentária e ajudar no controle do desenvolvimento, para reduzir ou redirecionar o esqueleto ou para extrusão dentes anteriores.

Estudos citam ainda blocos de mordida usados como um componente de aparelhos ortodônticos, os chamados Bite Blocks, que vão ajudar a corrigir problemas de MA esquelética, pode-se citar ainda conforme estudos feitos o Binator de Balters fechado que é tido como uma espécie de ativador, bionatores ou reguladores e é eficaz para o crescimento da dentição mista, sendo útil em casos de interposição lingual ou sucção. Para o uso de aparelho deve-se analisar caso a caso e combinar técnicas para um melhor resultado estético e funcional ${ }^{18,19,21}$. 
Por fim, em casos mais graves viu-se que o manejo cirúrgico é a técnica de correção que busca reorganizar a dentição e há ainda as glossectomias para correção de língua. E toda ação deste tipo deve primar pelo cuidado e em situações onde seja necessário o reposicionamento superior da maxila a cirurgia ortognática deve ser efetuada ${ }^{4,9}$.

Bona et al. (2016) ${ }^{17}$ tratam que a MAA deve ser encarada como um desafio a ser enfrentado rotineiramente na clínica odontológica, haja vista que a ausência de trespasse vertical permite a interposição lingual e a mesma, em repouso ou em atividade, perpetua à máoclusão.

Os alertas para que o tratamento seja precoce se dá pelo fato de que se os casos de MAA não tratados deixarem sequelas mais graves o tratamento pode ser feito desde uma simples grade palatina a uma aparelhagem fixa; ou com uso de dispositivos recordatórios e impedidores até com cirurgia ortognática quando há comprometimento esquelético severo. Ressalta-se a importância de confiança no profissional que está a frente do tratamento para que se tenha sucesso neste, e englobando ainda uma terapia multidisciplinar ${ }^{13,16,18,20}$.

A terapia ortodôntica depende muito de caso a caso, vê-se que na Ortodontia busca sempre o melhor tratamento para cada situação em prol de desenvolver a melhor forma que afete menos o paciente, sendo cada vez mais eficaz, por isso são discutidas aqui as principais técnicas utilizadas para resolver problemas de MAA que afetam as funções dos dentes, e qual técnica adequada é mais fácil de ser resolvido o problema diagnosticado ${ }^{16,21}$.

Vale acrescer que a terapêutica miofuncional periodicamente ressurge como um método de tratamento. Mas atualmente não há evidência científica suporta que garante essa terapia como eficaz na correção de MA. Com base nos estudos feitos demonstra-se que há necessidade de abranger mais tais problemas, buscando efetivar medidas preventivas de cunho nacional, em prol de um melhor planejamento e com a efetivação e organização de programas e serviços públicos de saúde bucal, com efetivação de promoção a saúde e medidas profiláticas para evitar mais problemas de $\mathrm{MAA}^{13,21}$.

Para algumas situações a cirurgia ortognática representa a melhor e mais previsível forma de tratamento, mas de forma diversa há casos em que por meio de intrusão de molares e controle extrusivo de incisivos o tratamento será de sucesso. ${ }^{12}$ Por fim, Pinho (2011) destaca que a MAA em adultos foi melhorando com tratamento pós desenvolvimento da técnica 
cirúrgica que permitiu o reposicionamento tridimensional e estável dos maxilares com as osteotomias $^{20}$.

\section{Considerações Finais}

Diante do exposto, os estudos demonstraram que a MAA é dentre os problemas de oclusão o que gera mais problemas estéticos e funcionais, tanto na infância quando na vida adulta e ele irá afetar a qualidade de vida dos portadores desta má-oclusão.

O tratamento é imprescindível e necessário e por isso avaliar cada situação específica e sua etiologia são determinantes para o sucesso da terapia e cada tipo de tratamento observado será variado conforme cada situação diagnosticada, mas na maioria delas incentiva-se um tratamento com uma equipe multidisciplinar que contribuem para melhorar a qualidade de vida do paciente.

\section{Referências}

1. Greenlee GM, Huang GJ, Shih-Hsuan S, Chen J , Koepsell T, Hujoel P. Estabilidade de tratamento para ântero-mordida aberta má oclusão: Uma meta-análise . Am Jour Ort e Orto Fac. 2011, 139 (2).

2. Miotto MHMB, Cavalcante WS, Godoy LM, Campos DMKS, Barcellos LA. Prevalência de mordida aberta anterior associada a hábitos orais deletérios em crianças de 3 a 5 anos de Vitória -ES. Rev. CEFAC. 2014; 16(4):1303-1310.

3. Sobral MC, Habib FA. Nascimento AC. Vertical control in the Class III compensatory treatment. Dental Press J. Orthod. 2013, 18 (2): 141-159.

4. Artese A, Drummond S, Nascimento JM, Artese F. Critérios para o diagnóstico e tratamento estável da mordida aberta anterior. Dental Press J Orthod. 2011;16(3):136-61.

5. Oliveira AP. Tratamento da Mordida Aberta Anterior Na Fase de Dentadura Mista. Trabalho de Conclusão do Curso de Odontologia - Universidade Estadual de Londrina, Londrina, 2015.78f. 
6. Boeck E, Pizzol K, Barbosa E, Pires N, Lunardi N . Prevalência de má oclusão em crianças de 3 a 6 anos portadoras de hábito de sucção de dedo e/ou chupeta. Rev Odontol UNESP. 2013; 42(2): 110-116.

7. Rocha AS, Rodrigues AA. Tratamento da Mordida Aberta e Utilização de Mini-Implante na Ortodontia: Uma Revisão Integrativa. Rev Cient Multi Núcleo do Conhecimento. 2017, 7(2): $235-244$.

8. Sousa R, Ribeiro G, Targino R, Martins C, Granville-Garcia AF, Paiva S. Prevalenc and Associated Factors for the Development of Anterior Open Bite and Posterior Crossbite in the Primary Dentition. Braz Dent Jou. 2014, 25(4): 336-342.

9. Aragón ML, Bichara LM, Flores MC, Almeida G, Normando D. Efficiency of compensatory orthodontic treatment of mild Class III malocclusion with two different bracket systems. Dental Press J. Orthod. 2017; 22(6): 49-55.

10. Farfán RL, Mattos MA, Soldevilla GL.Degradación de la fuerza de los elásticos intermaxilares de látex y no látex. Int. J. Odontostomat., 2017, 11(3):363-368.

11. Rahpeyma A, Khajehahmadi S. Force relaxation of $3 / 16$ inch heavy orthodontic latex elastics used in maxillofacial trauma in simulated jaw fracture situation. Dent Hyp, 2014, 5(1):146-149.

12. Kawabata E., et al. Color changes of esthetic orthodontic ligatures evaluated by orthodontists and patients: a clinical study. Dental Press J. Orthod.. 2016, 21 (5): 53-57.

13. Bruggemann R, Barbieri Tomé S, Tonetto A, Heizemann G, Burmann P, Beck D, Ruschel G. Mordida aberta anterior: Etiologia e tratamento. Ver Sal Integ. 2013, 6 (1): 11-12.

14. Moimaz S, Rocha N, Garbin A, Saliba O. A influência da prática do aleitamento materno na aquisição de hábi- tos de sucção não nutritivos e prevenção de oclusopatias. Rev Odontol da UNESP, Marília, 2013, 42 (1):31-36.

15. Melink S, Vagner M, Hocevar B. Posterior crossbite in the deciduous dentition period, its relation with sucking habits, irregular orofacial functions, and otolaryngological findings. Am J Orthod Dentofacial Orthop. 2010, 138 (3): 32-40.

16. Rochelle I, Silva E, Tagliaferro A, Meneguim M, Nobílo. Krunislave A, Ambrosano. G. Amamentação, hábitos bucais deletérios e oclusopatias em crianças de cinco anos de idade em São Pedro, SP. Dental Press J. Orthod. 2010, 15 (2).

17. Bona AP, Moreira K, Tedesco T, Imparato J, Ferreira B, Reis J. Abordagem multidisciplinar de mordida aberta anterior associada à sucção digital: caso clínico. Rev. Assoc. Paul. Cir. Dent. 2016; 70(1): 58-63. 
18. Pacheco AB, Da Silva AMT, Mezzomo CL, Berwig LC, Neu AP. Relação da respiração oral e hábitos de sucção não-nutritiva com alterações do sistema estomatognático. Rev CEFAC 2012;14(2):281-289.

19. Romero CC, Scavone-Junior H, Garib DG, Cotrim-Ferreira FA, Ferreira RI. Breastfeeding and non-nutritive sucking patterns related to the prevalence of anterior open bite in primary dentition. J Appl Oral Scielo 2011;19(2):161-8

20. Pinho T. A Ortodontia Intercetiva nas Deformidades Dento-Maxilares. Nascer e Crescer. 2011; 20(3): 192-196.

21. Baek M, Choi Y, Yu H, Lee K, Kwak J, Park Y. Long-term stability of anterior open-bite treatment by intrusion of maxillary posterior teeth. American Journal of Orthodontics and Dentofacial Orthopedics. V. 138, N. 4 p. 396, 2010.

\section{Como citar este artigo (Formato ABNT):}

VIEIRA, Islana Cleia Carvalho; VIEIRA, Idalécio Carvalho; AZEREDO, Morgana Souza de; JARDIM, Lauren Stephanie Vieira; PRADO, Jônatas Pereira do; REIS, Jade Alexandre Belo; LESSA, Anne Maria Guimarães; Tratamento de mordida aberta anterior: Uma revisão de literatura. Id on Line Rev.Mult. Psic., 2018, vol.12, n.42, p. 984-995, 2018. ISSN: 1981-1179.

Recebido: 24/10/2018;

Aceito: $29 / 10 / 2018$ 